\title{
Aberration-Corrected STEM for Elemental Mapping
}

\author{
N. Dellby, O.L. Krivanek, M. Murfitt, P.D. Nellist and Z.S. Szilagyi \\ Nion Co., $11028^{\text {th }}$ St., Kirkland, WA 98033, USA
}

Aberration correctors for scanning transmission electron microscopes (STEMs) now readily produce sub- $\AA$ resolution high-angle annular dark field (HAADF) images $[1,2]$. They also make possible substantially increased currents in atom-sized probes. The improvement in the probe current brings forth the day when signals with cross-sections smaller than high-angle elastic scattering will be able to routinely produce atomic-resolution information. The foremost among such signals is inner-shell loss inelastic scattering, probed by electron energy-loss spectroscopy (EELS). This paper briefly reviews our efforts towards the production of atomic-resolution EELS elemental maps.

In the absence of probe-enlarging instabilities, the current in an electron probe is given by:

$$
\mathrm{I}=\left(\mathrm{d}^{2}-\mathrm{d}^{2}{ }_{\text {aberr }}\right) \mathrm{B} \lambda^{2} / \mathrm{d}^{2}{ }_{\text {aberr }}
$$

where $\mathrm{d}$ is the total probe size which is partly due to aberrations and partly due to the finite demagnification of the electron source, $d_{\text {aberr }}$ is the aberration-determined probe size (i.e., the probe size in the limit of infinite source demagnification), B the brightness of the electron source and $\lambda$ the electron wavelength. The equation is only valid in the limit of the gun aberrations remaining unimportant, i.e. for probe currents less than a few $\mathrm{nA}$ with a cold field emission gun. It shows that as $d$ is lowered towards $d_{\text {aberr }}$ (by demagnifying the source more), the current in the probe falls off precipitously, and also that at larger currents where $d \gg d_{\text {aberr }}$, I is proportional to $\left(d / d_{\text {aberr }}\right)^{2}$.

Fig. 1 shows the expected probe current for a $200 \mathrm{kV}$ cold field emission STEM that is limited by 8fold astigmatism $\mathrm{C}_{7,8}$ and also chromatic aberration $\mathrm{C}_{\mathrm{c}}$ to $0.5 \AA$ probe size, compared to an existing uncorrected $200 \mathrm{kV}$ Schottky gun STEM. For probe sizes in the 1-2 $\AA$ range, the aberrationcorrected STEM will give probe currents that are about two orders of magnitude bigger than in present-day STEMs, and hence will be able to largely compensate for the fact that the cross-sections for inner-shell loss scattering are typically 2 to 4 orders of magnitude weaker than for elastic high angle scattering.

Unlike an imaging filter, the STEM does not need to focus the scattered electrons into a magnified image and can therefore form EELS images and elemental maps that are not made blurry by chromatic aberration. The increased probe current should therefore make atomic-resolution EELS imaging readily possible. However, such performance will place increased demands on the characteristics of the EELS system. In particular, the spectrometer will need to be able to accept the majority of inner shell loss electrons without a loss of energy resolution. It will also need to acquire spectra with high efficiency and high speed, especially for mapping applications.

In practice, the above demands mean that 1) the spectrometer coupling optics needs to be designed with as much care as the probe-forming optics, 2) the aberration performance of the spectrometer needs to be improved, and 3) fast and efficient CCDs capable of acquiring 1000 or more spectra per second will need to be used. The sensor efficiency has been greatly improved by the CCD-based Gatan Enfina spectrometer, but the other issues remain pressing.

Improved coupling optics is especially needed by the corrected $100 \mathrm{kV} \mathrm{VG} \mathrm{STEM} \mathrm{with} \mathrm{no} \mathrm{post-}$ specimen lenses, which gives only a factor of 3x typical post-sample angular compression. We have therefore developed a quadrupole coupling module (QCM) incorporating 4 quadrupoles and 4 octupoles for this instrument. The QCM is able to increase the angular compression into an Enfinatype energy loss spectrometer, make the height of the spectrometer entrance crossover adjustable, 
and correct all geometrical third order EELS aberrations. It starts just below the level of the VG lid, and it is followed by a detector module and the energy-loss spectrometer.

Fig. 2 shows the electron trajectories through the QCM. The scattered electrons only enter the quadrupole coupling module (QCM) after $240 \mathrm{~mm}$ of travel through the top-entry specimen cartridge, the post-sample deflection (Grigson) coils and the pre-QCM HAADF detector. Even with the $3 \mathrm{x}$ angular compression due to the post-field of the objective lens, an electron beam encompassing electrons leaving the sample at up to $30 \mathrm{mrad}$ to the optic axis is $5 \mathrm{~mm}$ wide as it enters the QCM. This is unusually high in precision electron optics, and illustrates the challenge posed by the weak post-sample compression of the VG microscopes. The QCM increases the angular compression to about 7x, but at the entrance aperture of the spectrometer situated $130 \mathrm{~mm}$ in front of the prism, the beam is again $5 \mathrm{~mm}$ in diameter. Sub-eV resolution then requires that the spectrometer's third order aberrations be eliminated. This is accomplished using the 4 octupoles, two of which act in the dispersion plane of the prism (a-type), and two of which are "skew" octupoles (b-type). One of each type is placed in the center of the QCM, where the beam is highly elongated in the dispersion $(\mathrm{X})$ direction, the others are placed after the QCM's quadrupoles and act on a round beam.

A similar quadrupole coupling module will be used on the optimized aberration-corrected STEM column we are now building. Because the QCM can create a virtual EELS entrance crossover in front of itself, it allows the column to be shortened by tens of $\mathrm{cm}$ compared to the length that would be required if round lenses were used for coupling to a standard post-column spectrometer. The QCM's ability to correct third order EELS aberrations together with the much higher post-specimen compression factors of the new column will guarantee good energy resolution even at collection angles of $50 \mathrm{mrad}$ and higher. These features are expected to optimize the EELS performance of the new column, and to lead to routine EELS elemental mapping with $2 \AA$ or better spatial resolution.

[1] P.E. Batson, N. Dellby and O.L. Krivanek, Nature 418 (2002) 617.

[2] S.J. Pennycook et al., these proceedings.

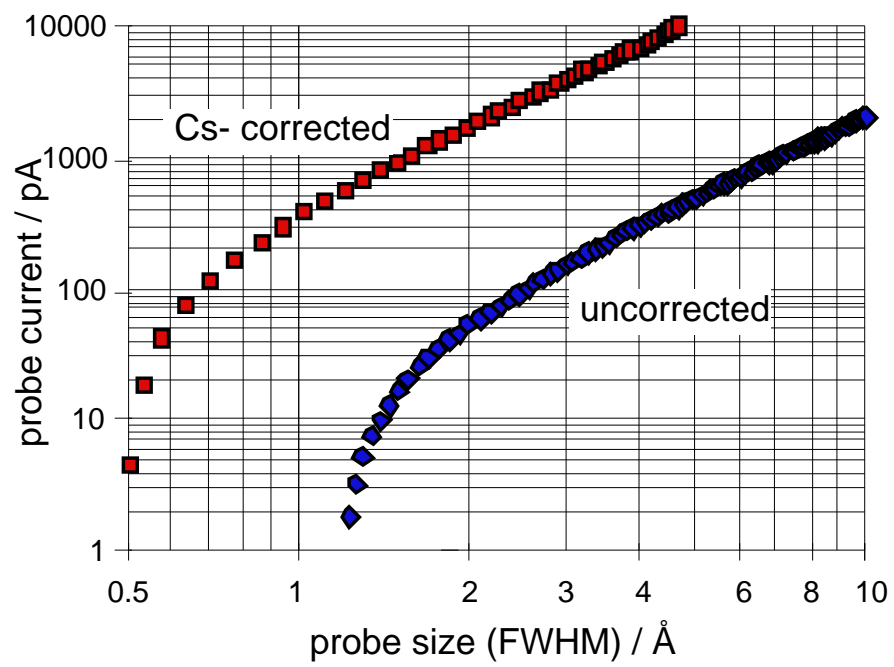

Fig. 1. Probe current vs. probe size. $\mathrm{C}_{\mathrm{s}}$-corrected: 200 $\mathrm{keV}, \mathrm{C}_{3} / \mathrm{C}_{5}$ corrected, $\mathrm{C}_{7,8}=10 \mathrm{~cm}$, cold field emission gun. Uncorrected: $\mathrm{C}_{\mathrm{s}}=0.5 \mathrm{~mm}$, Schottky gun.

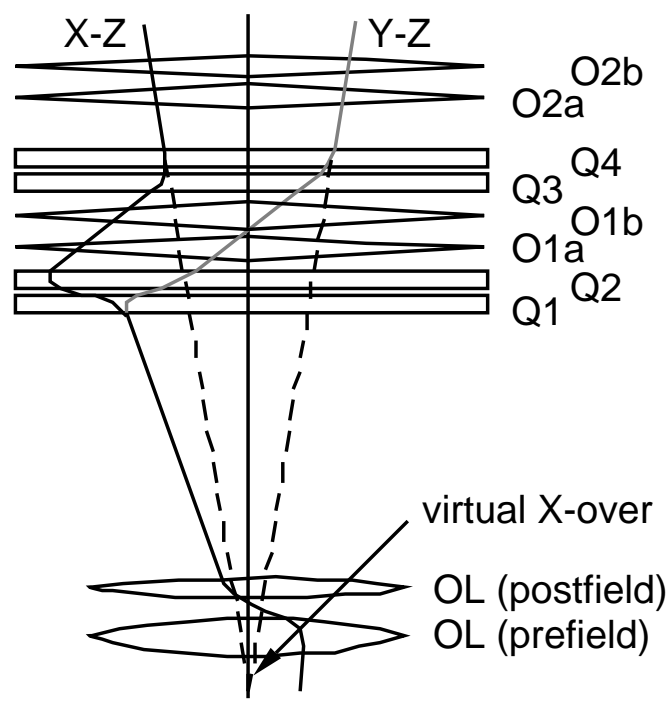

Fig. 2. Trajectories through the quadrupoleoctupole EELS coupling module. The objective lens and the EELS virtual crossover are also shown. 